\title{
On the Queue Tail Asymptotics for General Multifractal Traffic
}

\author{
Sándor Molnár ${ }^{1}$, Trang Dinh Dang ${ }^{1}$, and István Maricza ${ }^{1}$ \\ High Speed Networks Laboratory, \\ Dept. of Telecommunications and Telematics, \\ Budapest University of Technology and Economics \\ H-1117, Magyar tudósok körútja 2, Budapest, Hungary \\ Tel: (361) 463 3889, Fax: (361) 4633107 \\ \{molnar, trang, maricza\}@ttt-atm.ttt.bme.hu
}

\begin{abstract}
The tail asymptotics in an infinite capacity single server queue serviced at a constant rate and driven by general multifractal input process is presented. It has been shown that in the important subcase of the monofractal Fractional Brownian Motion (FBM) input traffic our result gives the well-known Weibullian tail. Practical engineering applications and validation of the results based on the analysis of measured network traffic have also been presented.
\end{abstract}

\section{Introduction}

Teletraffic research papers have reported the high variability and burstiness nature of network traffic in several LAN/WAN environments in the last decade. Moreover, it seems that most of the measured network traffic exhibits properties of scale invariance. It means that within a range of scales no characteristic dominant scale can be identified and some statistical properties within this range are not changing. This remarkable scaling phenomenon called for the fractal modeling of the investigated LAN/WAN traffic 2120|919].

In the fractal modeling framework long-range dependence (LRD) and selfsimilarity have been analyzed intensively, and a number of studies is focused on how to detect accurately the LRD property and how to estimate the Hurst parameter 32. LRD is revealed by the power law decay of the autocorrelation function at large lags, i.e., $r(k) \sim c|k|^{2 H-2}, k \rightarrow \infty, H \in(0.5,1)$, where $c$ is a constant [3]. The degree of this slow decay is determined by the Hurst parameter $(H)$.

A large group of traffic models (Fractional Brownian Motion (FBM) models, FARIMA models, Cox's M/G/ $\infty$ models, on/off models, etc.) to capture LRD and self-similar properties has also been developed [16]. Among these models the FBM [17] was found to be a popular parsimonious and tractable model of traffic aggregation [412]. The performance implications of the fractal property are also addressed in a series of studies [877.

After a number of new measurements and deeper analysis of network traffic it was discovered that the LAN/WAN traffic has a more complex scaling behaviour

E. Gregori et al. (Eds.): NETWORKING 2002, LNCS 2345, pp. 105-116 2002.

(C) Springer-Verlag Berlin Heidelberg 2002 
which cannot be described by LRD and self-similarity 219]. More precisely, it has been found that aggregate network traffic is asymptotically self-similar over time scales of the order of a few hundreds of milliseconds and above but it exhibits multifractal scaling below this time scale [9]. It has been also pointed out that the transition from the multifractal to self-similar scaling occurs around time scales of a typical packet round-trip time in the network 9]. However, some studies showed that multifractal scaling can also be present even at large time scales [15]. Therefore the monofractal traffic models (e.g. FBM) are inadequate to characterize the network traffic and multifractal traffic models with a much more flexible rule for the scaling law seem to be needed, especially for some WAN environments. Multifractal models can allow a compact description of a complex scaling behavior and it can also capture the non-Gaussian character of network traffic. Multifractal models imply the non-redundant scaling behavior of moments of many orders. The physical explanations and engineering implications are also addressed in several papers, e.g. [9].

A stochastic process $X(t)$ is called multifractal [13] if it has stationary increments and satisfies

$$
\mathbb{E}\left[|X(t)|^{q}\right]=c(q) t^{\tau(q)+1}
$$

for some positive $q$, where $\tau(q)$ is called the scaling function of multifractality and $c(q)$ is independent of $t$. An easy consequence of this definition is that $\tau(q)$ is a concave function [13. If the scaling function $\tau(q)$ is a linear function of $q$ the process is called monofractal. Multifractality is thus defined as a global property of the process moments. The definition is very general and it covers a very large class of processes. Multifractal processes are also called processes with scaling property.

From a practical point of view queueing analysis of fractal traffic is a very important issue for network dimensioning and management. Therefore the study of queueing systems with fractal traffic input is a challenge in queueing theory. In the recent years the performance of queues with LRD or self-similar input has been deeply analyzed. A collection of studies has proven that the FBM based models have a tail queue distribution that decays asymptotically like a Weibullian law, i.e., $\mathbf{P}[Q>b] \simeq \exp \left(-\delta b^{2-2 H}\right)$, where $\delta$ is a positive constant that depends on the service rate of the queue 176 . This important result shows that queues with FBM input $(H>1 / 2)$ have a much slower decay than that of the exponential.

However, there is a lack of queueing results available in the cases when the input traffic has a more complex scaling behaviour. Especially, queueing systems with multifractal input are an undiscovered field and only a few results have been published in the literature. Véhel et al. 22] suggested a cascade model for TCP traffic based on the retransmission and congestion avoidance mechanisms with no performance analysis. Riedi et al. [19 18] developed a multiscale queueing analysis in the case of tree-based multiscale input models. Gao et al. simulated queues fed by multiplicative multifractal processes in [10] but provided no analytical results. In contrast to these results we consider general multifractal 
process without any restrictions and derive analytical results for the queue tail asymptotics.

Our aim is to contribute to the queueing theory of multifractal queues and also to the traffic engineering implications. In this paper we present a novel analysis of multifractal queues including the tail asymptotics, special cases, and practical applications.

\section{Queueing Model}

We consider a simple queueing model: a single server queue in continuous time, the serving principle for offered work is defined to be FIFO (First In, First Out), the queue has infinite buffer and constant service rate $s$. Denote by $X(t)$ the total size of work arriving to the queue from time instant $-t$ in the past up to this moment, time instant 0 . The so called workload process $W(t)$ is the total amount of work stored in the buffer in time interval $(-t, 0)$, i.e.,

$$
W(t)=X(t)-s t
$$

Our interest, however, is the current buffer length of the queue, denoted by $Q$. This is the queue length in the equilibrium state of the queue when the system has been running for a long time and the initial queue length has no influence. If this state of the system does exist, i.e., stationarity and ergodicity of the workload process hold, and the stability condition for the system is also satisfied, i.e., $\lim \sup _{t} \mathbb{E}[X(t)] / t<s$, then:

$$
Q=\sup _{t \geq 0} W(t)
$$

where $W(0)$ is assumed to be 0 . This equation is also referred to as Lindley's equation.

The input process $X(t)$ is considered as a general multifractal process which is defined by Eq. 1. This definition, presented by Mandelbrot et al. in [13, describes multifractal processes in terms of moments which leads to a more intuitive understanding of multifractality.

\section{Approximation for Queue Tail Probabilities}

We now state our main proposition:

Proposition 1. The probabilities for the queue tail asymptotic of a single queueing model with general multifractal input is accurately approximated by:

$$
\log (\mathbf{P}[Q>b]) \approx \min _{q>0} \log \left\{c(q) \frac{\left[\frac{b \tau_{0}(q)}{s\left(q-\tau_{0}(q)\right)}\right]^{\tau_{0}(q)}}{\left[\frac{b q}{q-\tau_{0}(q)}\right]^{q}}\right\}, \quad b \text { large }
$$

where $\tau_{0}(q):=\tau(q)+1$. The scaling function $\tau(q)$ and $c(q)$ are the functions which define the multifractal input process. 


\section{Proof}

Using Lindley's equation the tail probabilities of queue length can be rewritten of the form: $\mathbf{P}[Q>b]=\mathbf{P}\left[\sup _{t \geq 0} W(t)>b\right]$. First let consider the quantity $\mathbf{P}[W(t)>b]:$

Replacing $W(t)$ by Eq. 2 we have

$$
\begin{aligned}
\mathbf{P}[W(t)>b] & =\mathbf{P}[X(t)-s t>b] \\
& \leq \mathbf{P}[|X(t)|>b+s t] \\
& =\mathbf{P}\left[|X(t)|^{q}>(b+s t)^{q}\right], \quad \text { for arbitrary } q>0 \\
& \leq \frac{E\left[X(t)^{q}\right]}{(b+s t)^{q}}, \quad \text { using Markov's inequality. }
\end{aligned}
$$

Since the input process is multifractal defined by Eq. 1 then:

$$
\begin{gathered}
\mathbf{P}[W(t)>b] \leq \frac{c(q) t^{\tau_{0}(q)}}{(b+s t)^{q}} \\
\Rightarrow \sup _{t \geq 0} \mathbf{P}[W(t)>b] \leq \sup _{t \geq 0} \frac{c(q) t^{\tau_{0}(q)}}{(b+s t)^{q}}=: \sup _{t \geq 0} f(t) .
\end{gathered}
$$

The straightforward derivation of $f(t)$ shows that it has a maximal value at $t=\frac{b \tau_{0}(q)}{s\left[q-\tau_{0}(q)\right]}>0$. Therefore

$$
\begin{gathered}
\sup _{t \geq 0} \mathbf{P}[W(t)>b] \leq \sup _{t \geq 0} f(t)=c(q) \frac{\left[\frac{b \tau_{0}(q)}{s\left(q-\tau_{0}(q)\right)}\right]^{\tau_{0}(q)}}{\left[\frac{b q}{q-\tau_{0}(q)}\right]^{q}} \\
\Rightarrow \log \left(\sup _{t \geq 0} \mathbf{P}[W(t)>b]\right) \leq \log \left(c(q) \frac{\left[\frac{b \tau_{0}(q)}{s\left(q-\tau_{0}(q)\right)}\right]^{\tau_{0}(q)}}{\left[\frac{b q}{q-\tau_{0}(q)}\right]^{q}}\right), \quad \text { for arbitrary } q>0 \\
\Rightarrow \log \left(\sup _{t \geq 0} \mathbf{P}[W(t)>b]\right) \leq \min _{q>0} \log \left(c(q) \frac{\left[\frac{b \tau_{0}(q)}{s\left(q-\tau_{0}(q)\right)}\right]^{\tau_{0}(q)}}{\left[\frac{b q}{q-\tau_{0}(q)}\right]^{q}}\right)
\end{gathered}
$$

For a large class of stochastic processes (including FBM) the following limit holds [11:

$$
\lim _{b \rightarrow \infty} \frac{\log (\mathbf{P}[Q>b])}{\log \left(\sup _{t \geq 0} \mathbf{P}[W(t)>b]\right)}=1 .
$$

In addition,

$$
\log (\mathbf{P}[Q>b]) \geq \log \left(\sup _{t \geq 0} \mathbf{P}[W(t)>b]\right),
$$

then the right-hand side of Eq. [ 8 is a upper bound of a lower bound on $\log (\mathbf{P}[Q>$ $b]$ ). The used inequalities in Eq. 10 and Eq. [6 become tight for finite large $b$. 
Thus our approximation for the queue tail asymptotics is the following:

$$
\log (\mathbf{P}[Q>b]) \approx \min _{q>0} \log \left(c(q) \frac{\left[\frac{b \tau_{0}(q)}{s\left(q-\tau_{0}(q)\right)}\right]^{\tau_{0}(q)}}{\left[\frac{b q}{q-\tau_{0}(q)}\right]^{q}}\right), \quad b \text { large. }
$$

For positive multifractal processes, i.e. $X(t)>0$, Eq. [5] is an equality. In addition, the approximation in Eq. 10 and the inequality in Eq. 6 turn to be more accurate approximations as $b$ tends to infinity. Thus the presented approximation is supposed to be asymptotically tight. The tightness and accuracy of the approximation is also experimentally investigated in Section V.

Considering the formula in Eq. 4 we see that it has an implicit form and just the given form of the functions $c(q)$ and $\tau(q)$ can provide the final result. The reason behind this is that the definition for the class of multifractal processes gives no restrictions for the functions $c(q)$ and $\tau(q)$ (beyond that $\tau(q)$ is concave). Our conjecture is that the analysis of queueing systems with general multifractal input may produce some similar general results. It means that there is no general queueing behaviour for these systems as the Weibullian decay in the case of Gaussian self-similar processes [17]. An actual multifractal model will determine, for example, the queue length probabilities of the system.

\section{Applications}

\subsection{Fractional Brownian Motion}

As a simple application first we consider a monofractal Gaussian process, called Fractional Brownian Motion (FBM). FBM is self-similar which is a simple case of monofractality and it is also Gaussian. The increment process of FBM is called Fractional Gaussian Noise (FGN). Queueing analysis of a single queue with FBM input is first presented by Norros 17] which showed the Weibullian decay for the asymptotic tail behaviour, i.e., $\mathbf{P}[X>x] \sim \exp \left(-\gamma x^{\beta}\right)$ with $\beta \leq 1$. This result is also justified by Large Deviation techniques in [6]. Applying this input process model to our formula should show its use and robustness when comparing to these available results.

First we prove that any Gaussian process with scaling property is in the class of monofractal processes. Furthermore we give the explicit forms for $\tau(q)$ and $c(q)$.

Consider the following lemma:

Lemma 1. A Gaussian process with scaling property is monofractal with parameters

$$
\left\{\begin{array}{l}
\tau(q)=\frac{q}{2}[\tau(2)+1]-1 \\
c(q)=\frac{[2 c(2)]^{q / 2}}{\sqrt{\pi}} \Gamma\left(\frac{q+1}{2}\right),
\end{array}\right.
$$

where $\Gamma(\cdot)$ denotes the Gamma function, $\Gamma(z)=\int_{0}^{+\infty} x^{z-1} \exp ^{-x} \mathrm{~d} x, z>0$.

The proof of this Lemma is provided in [5]. 
Turning back to our case of FBM with $c(2)=1$ and $\tau(2)=2 H-1$ where $H$ is referred to as the Hurst parameter, we have

$$
\left\{\begin{array}{l}
\tau(q)=q H-1 \\
c(q)=\frac{2^{q / 2}}{\sqrt{\pi}} \Gamma\left(\frac{q+1}{2}\right)
\end{array}\right.
$$

Insert these two functions into our formula in Eq, 4 we get

$$
\log (\mathbf{P}[Q>b]) \approx \log \left(\min _{q>0}\left\{\frac{2^{q / 2}}{\sqrt{\pi}} \Gamma\left(\frac{q+1}{2}\right) \frac{\left(\frac{b H}{s(1-H)}\right)^{q H}}{\left(\frac{b}{1-H}\right)^{q}}\right\}\right)=: \log \left(\min _{q>o} g(q)\right) .
$$

The minimum value of the $g(q)$ for $q>0$ function can be easily determined by taking its derivatives. The result is the following:

$$
\log (\mathbf{P}[Q>b]) \approx \log \left(\min _{q>o} g(q)\right)=\log \left(\frac{1}{\sqrt{\pi}} \frac{\Gamma\left(\Psi^{-1}(\log K)\right)}{K^{\Psi^{-1}(\log K)-1 / 2}}\right)=: \log \left(T_{F B M}(H, s, b)\right)
$$

where $K=K(H, s, b)=\frac{1}{2} b^{2(1-H)} s^{2 H}(1-H)^{-2(1-H)} H^{-2 H}, \Psi(\cdot)$ is the digamma function, $\Psi(x)=\frac{\mathrm{d}}{\mathrm{d} x} \log \Gamma(x)=\frac{\Gamma^{\prime}(x)}{\Gamma(x)}$, and $\Psi^{-1}(\cdot)$ denotes the inverse function of $\Psi(\cdot)$.
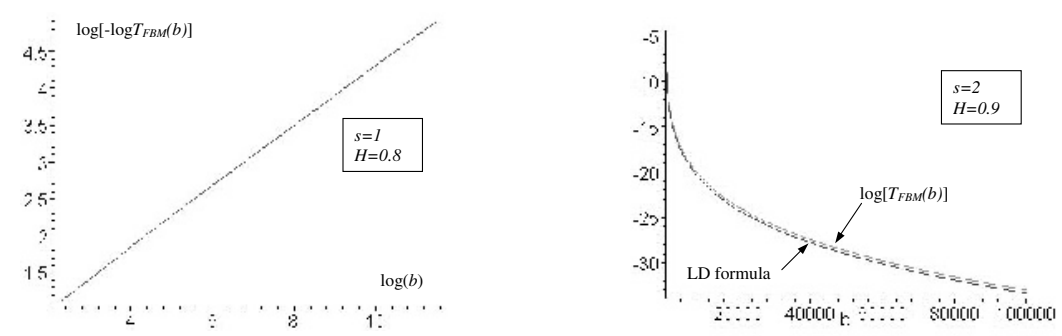

Fig. 1. By setting fixed values for $H$ and $s$, Fig. 2. Our approximation compared to the line in the $\log$-log plot of $-\log T_{F B M}(b)$ the Large Deviation technique result. versus $b$ clearly shows the Weibullian decay for $T_{F B M}(H, s, b)$.

The $T_{F B M}(H, s, b)$ function is quite complex with the presence of Gamma, digamma, and its inverse function. However, we have quite a good approximation of $T_{F B M}(H, s, b)$ :

Proposition 2. The approximation

$$
\frac{1}{\sqrt{\pi}} \frac{\Gamma\left(\Psi^{-1}(\log x)\right)}{x^{\Psi^{-1}(\log x)-1 / 2}} \approx \exp (-x)
$$

holds for large $x, x>0$.

The proof and the precise sense of this approximation can be found in [5]. 
Applying this approximation we find that the queue tail for the FBM case satisfies:

$$
\log \left(T_{F B M}(H, s, b)\right) \approx-\frac{1}{2} b^{2(1-H)} s^{2 H}(1-H)^{-2(1-H)} H^{-2 H}, \quad b \text { large. }
$$

Eq. 13 shows the Weibullian decay of this queue which was first recognized and proven by Norros [17]. Numerical evaluations of the result are presented in Fig. 1 and Fig. 2. In Fig. [1] we fix the values of $H$ and $s$ and then calculate the values of the queue tail approximation $T_{F B M}(H, s, b)$ versus the queue size $b$ and then plot it in the log-log scale. The linearity of the plot also demonstrates the Weibullian decay.

Now we compare our result to the result obtained by Duffield and O'Connell. The asymptotic formula for queue tail probabilities provided by Large Deviation technique presented in [6] is

$$
\begin{aligned}
& \lim _{b \rightarrow \infty} b^{-2(1-H)} \log \mathbf{P}[Q>b]=-\inf _{c>0} c^{-2(1-H)} \frac{(c+s)^{2}}{2} \\
\Leftrightarrow & \log \mathbf{P}[Q>b] \rightarrow-\frac{1}{2} b^{2(1-H)} s^{2 H}(1-H)^{-2(1-H)} H^{-2 H}, \quad \text { as } b \rightarrow \infty,
\end{aligned}
$$

where $s$ also denotes the service rate. Therefore we can conclude that our approximation yields the Large Deviation result, see Eq. 13 and Eq. 14. The two results are depicted in Fig. 2 and we can see that the plots almost coincide for all calculated values of the queue size.

Our conclusions can be summarized in two main points: (i) the asymptotic tail approximation for the case of FBM has Weibullian decay; (ii) this result is also consistent with the formula presented by Norros [17] and by Duffield et al. with Large Deviation technique [6].

In the case of $H=1 / 2$ (Brownian Motion) the above formula results in $\log \mathbf{P}[Q>b] \approx-2 s b / \sigma^{2}$ where $\sigma^{2}$ denotes the variance of the process, which is in agreement with the queueing formula known from the theory of Gaussian processes [14,6].

\subsection{Practical Solutions}

We show here the practical use of the formula. Assume that we are interested in the behaviour of the tail of the steady-state buffer occupancy (queue length) distribution at a specific multiplexer in our network. The first step should be the fine resolution measurements of the input process. We also assume that the input process exhibits multifractal scaling properties. Then the scaling function $\tau(q)$ and the function $c(q)$ can be estimated from the collected data for some available parameters $q>0$. We emphasize the importance of the function $c(q)$ as the quantity factor of multifractal processes which is sometimes neglected in a number of studies dealing with multiscaling properties of the high-speed network traffic. The scaling function $\tau(q)$ defines only the quality of multiscaling and it is not enough for the description of a multifractal model and therefore for the analysis of queueing models with multifractal input processes. 
Now we suggest two practical methods for the approximation of the queue tail distribution:

1. Given the service rate $s$ and the two sets $\{c(q)\}$ and $\{\tau(q)\}$, using Eq. 4 the approximation of $\log (\mathbf{P}[Q>b])$ can be computed for each value of $b$. This method is very simple but it is the more useful from network planning and capacity dimensioning point of view since we are only interested in some values of the tail probabilities. We mainly focus on the practical use of this method in this study.

2. The input process is fitted to a multifractal model. The two measured sets of $c(q)$ and $\tau(q)$ are fitted by $\tilde{c}(q)$ and $\tilde{\tau}(q)$. Then the analysis of the Eq. 4 with these functions can result in simple closed form of the queue tail probabilities. We use this method when studying the queue tail behaviour of a multifractal model.

\section{Queueing Analysis}

In this section we show the validation for the mentioned practical solution presented above by the queueing analysis of some real traffic traces. We also provide a simple method for estimation of multiscaling functions $c(q)$ and $\tau(q)$.

\subsection{Simple Method for Multiscaling Functions Estimation}

The full description of a multifractal model involves both $c(q)$ and the scaling function $\tau(q)$. We present here a simple method for testing of scaling properties and also for the estimation of these functions.

The definition of multifractal processes (Eq. 1) claims the stationarity condition for the increments. Therefore it is easy to verify the following relation for the moments of the increments: $\mathbb{E}\left[\left|Z^{(\triangle t)}\right|^{q}\right]=c(q)(\triangle t)^{\tau(q)+1}=c(q)(\triangle t)^{\tau_{0}(q)}, q>0$, where $Z^{(\triangle t)}$ denotes the increment process of time sample $\triangle t$. Thus $\mathbb{E}\left[\left|Z^{(m \triangle t)}\right|^{q}\right]$ $=c(q)(m \triangle t)^{\tau_{0}(q)}, q>0$ also holds for $m=1,2, \ldots$

Choose $\triangle t$ as the time unit, then

$$
\log \mathbb{E}\left[\left|Z^{(m)}\right|^{q}\right]=\tau_{0}(q) \log m+\log c(q), \quad q>0 .
$$

Based on this property, the method is the following: Given a data series of a process increments $Z_{1}, Z_{2}, \ldots, Z_{n}$ and define its corresponding real aggregated sequence $\left\{Z^{(m)}\right\}$ of the aggregation level $m$ by

$$
Z_{k}^{(m)}=Z_{(k-1) m+1}+Z_{(k-1) m+2}+\ldots+Z_{k m}, \quad k, m=1,2, \ldots
$$

If the sequence $\left\{Z_{k}\right\}$ has scaling property then the plot of absolute moments $\mathbb{E}\left[\left|Z^{(m)}\right|^{q}\right]$ versus $m$ on a log-log plot should be a straight line due to Eq. 15] The slope of the straight line provides the estimate of $\tau_{0}(q)$ and the intercept is the value for $\log c(q)$. The illustration of the method can be seen in Fig. 3 ,

Note that we have no need to estimate $c(q)$ and $\tau_{0}(q)$ for all positive value of $q$, which is an impossible task. In fact, the largest value of $q$ we should considered depends on the interested finite queue length of the involved queue length probability, see below. 


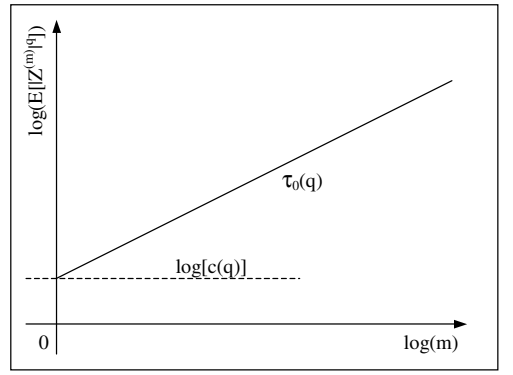

Fig. 3. A simple method for scaling test and the estimation of $c(q)$ and the scaling function $\tau(q)$.

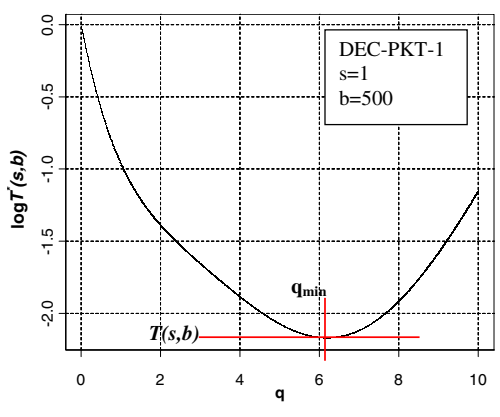

Fig. 4. Theoretical queue tail probability at each value of queue size $b$ is the minimum of $\log T^{*}(s, b)$.

\subsection{Analysis Results}

Our results have been first validated by simulation of multifractal cascades [5]. We have also carried out analysis of several measured IP packet arrival traffic traces (DEC-PKT-1, DEC-PKT-2, and DEC-PKT-3) obtained from the Internet Traffic Archive [1. In this paper we present only two typical cases, i.e., monofractal (DEC-PKT-2) and multifractal traffic (DEC-PKT-3). The analysis validates the use of our approximation in a single queue with constant service rate and general multifractal input.

Figure 5(a) shows the plot of absolute moments of the aggregated sets of the set DEC-PKT-3 versus the aggregation level in a log-log plot for some values of moment $q$. The linearity of the plots observed in the figure clearly indicates the scaling property of this data set. After applying the estimation method we presented in the previous subsection we get the two sets of estimated $\tau_{0}(q)$ and

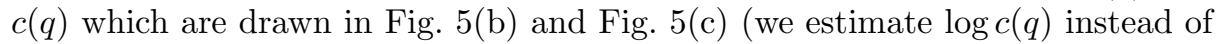
$c(q)$ ). The plot of the function $\tau_{0}(q)=\tau(q)+1$ is a concave curve which suggests the multifractal property of DEC-PKT-3.

We then make a comparison between our approximation and the queueing simulation of real data traces to validate the use of the formula in practice. The approximation for probabilities of queue tail presented in Proposition 1 can be rewritten in the form

$$
\begin{aligned}
\log \mathbf{P}[Q>b] & \approx \min _{q>0}\left\{\log c(q)+\tau_{0}(q) \log \frac{b \tau_{0}(q)}{s\left(q-\tau_{0}(q)\right)}-q \log \frac{b q}{q-\tau_{0}(q)}\right\} \\
& =: \min _{q>0}\left\{\log T^{*}(s, b)\right\}=T(s, b) .
\end{aligned}
$$

For the sake of calculation simplicity we choose the service rate such that $s=1$. The lower curve in Fig. 5 (d) shows the simulation result of the DEC-PFT-3 data set. Using Eq. [17] the value of the logarithmic tail probability at each concerned value of queue size $b$ is taken by the numerical minimization of $\log T^{*}(s, b)$ with the estimated sets $\{c(q)\}$ and $\left\{\tau_{0}(q)\right\}$. An example is shown in Fig. 4 . 


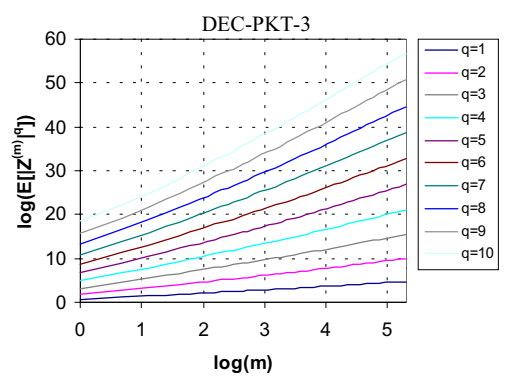

(a)

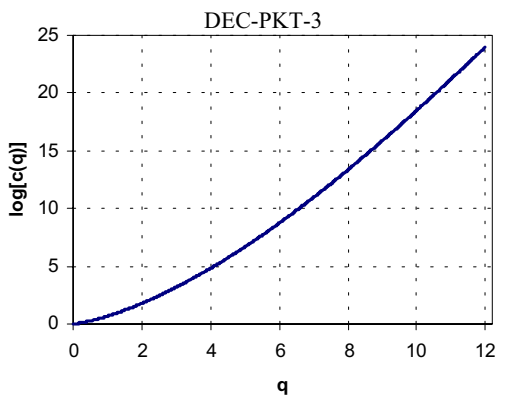

(c)

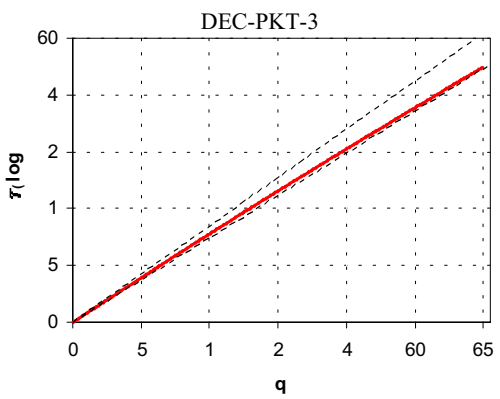

(b)

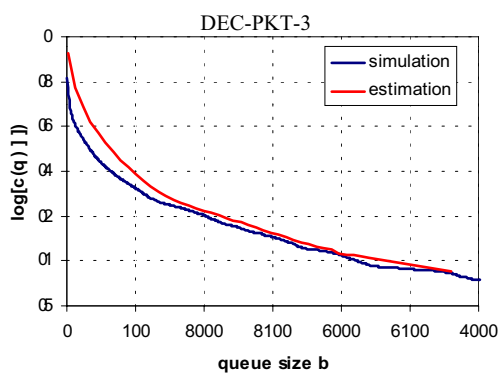

(d)

Fig. 5. Analysis results of the DEC-PKT-3 data set.

In addition, we do not need to plot $\log T^{*}(s, b)$ at each value of $q$ to find its minimum. A simple program routine can do it for all concerned value of $b$ at once. Our theoretical tail probabilities are on the upper curve in Fig. 5 As comparing with the simulation result which is seen in the same figure we found that it has the similar shape and becomes tight as $b$ increases. This validates our result.

We have performed the same analysis with an other data set DEC-PFK2. The results are summarized in Fig. 6. The DEC-PKT-2 data set, however, has the exact monofractal structure and can be well modelled by statistical selfsimilarity with Hurst parameter $H=0.8$. Our queueing model deals with general multifractal input so it also involves the case of monofractal processes. Thus it is not surprising that the analysis also provides the correct queueing results in this case.

\section{Conclusion}

In this paper we studied the queueing performance of a single server infinite capacity queue with a constant service rate fed by general multifractal input process. We have provided the following results: 


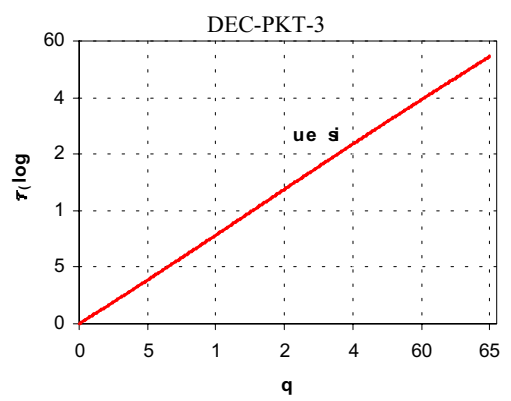

(a)

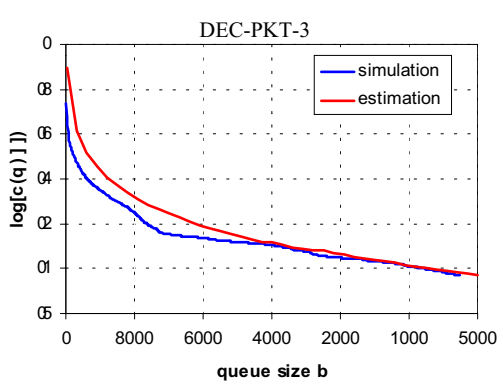

(b)

Fig. 6. Analysis results of the DEC-PKT-2 data set.

(i) We derived an asymptotic approximation of the steady-state queue length probabilities.

(ii) We showed that our results gives the well-known Weibullian queue tail in case of the monofractal Fractional Brownian Motion input process.

(iii) We proved that the class of Gaussian processes with scaling properties is limited to monofractal processes.

(iv) We demonstrated the practical applicability of our approximation and validated the method by queueing analysis of both multifractal and monofractal network traffic cases.

There are several interesting topics for further research. Based on the multifractal process characterization one of our goal is to build a multifractal traffic model parameterized by the multifractal functions. We also intend to carry out more multifractal analyses of measured LAN/WAN traffic with corresponding performance analysis.

\section{References}

1. The internet traffic archive. http://ita.ee.lbl.gov.

2. P. Abry and D. Veitch. Wavelet analysis of long range dependent traffic. IEEE Trans. Inform. Theory, 44(1):2-15, January 1998.

3. J. Beran. Statistics for Long-Memory Processes. Chapman \& Hall, One Penn Plaza, New York, NY 10119, 1995.

4. D. R. Cox. Statistics: An Appraisal, Proc. 50th Anniversary Conference, chapter Long-Range Dependence: A Review. Iowa State University Press, 1984.

5. T. D. Dang and S. Molnár. Queue asymptotics with general multifractal input. Technical report, Budapest University of Technology and Economics, July 2001.

6. N.G. Duffield and N. O'Connell. Large deviations and overflow probabilities for the general single-server queue, with applications. In Proc., Cam. Phil. Soc., volume 118, pages 363-374, 1994.

7. A. Erramilli, O. Narayan, A. L. Neidhardt, and I. Saniee. Performance impacts of multi-scaling in wide-area TCP/IP traffic. In Proc., IEEE INFOCOM 2000, volume 1, pages 352-359, Tel Aviv, Israel, 2000. 
8. A. Erramilli, O. Narayan, and W. Willinger. Experimental queueing analysis with long-range dependent packet traffic. IEEE/ACM Trans. on Networking, 4(2):209223, April 1996.

9. A. Feldmann, A. C. Gilbert, and W. Willinger. Data Networks as Cascades: Investigating the Multifractal Nature of Internet WAN Traffic. ACM Computer Communication Review, 28:42-55, September 1998.

10. J. Gao and I. Rubin. Multifractal modeling of counting processes of long-range dependent network traffic. In Proceedings SCS Advanced Simulation Technologies Conf., San Diego, CA, April 1999.

11. J. Hüsler and V. Piterbarg. Extremes of a certain class of Gaussian processes. Stochastic Process. Appl., 83:257-271, 1999.

12. T. G. Kurtz. Stochastic Networks: Theory and Applications, chapter Limit Theorems for Workload Input Models. Oxford University Press, 1996.

13. B. B. Mandelbrot, A. Fisher, and L. Calvet. A Multifractal Model of Asset Return. Yale University, 1997. Working Paper.

14. M. B. Marcus and L. A. Shepp. Sample behaviour of Gaussian processes. In Proceedings of the Sixth Berkeley Symposium, 1972.

15. S. Molnár and T. D. Dang. Scaling analysis of IP traffic components. In ITC Specialist Seminar on IP Traffic Measurement, Modeling and Management, Monterey, CA, USA, 18-20 September 2000.

16. S. Molnár and A. Vidács. On Modeling and Shaping Self-Similar ATM Traffic. In 15th International Teletraffic Congress, Washington, DC, USA, June 1997.

17. I. Norros. A storage model with self-similar input. Queueing Systems, 16:387-396, 1994.

18. V. J. Ribeiro, R. H. Riedi, M. S. Crouse, and R. G. Baraniuk. Multiscale queuing analysis of long-range-dependent network traffic. In Proceedings of IEEE INFOCOM 2000, Tel Aviv, Israel, March 2000.

19. R. H. Riedi, M. S. Crouse, V. J. Ribeiro, and R. G. Baraniuk. A multifractal wavelet model with application to network traffic. IEEE Trans. Inform. Theory, 45(3):992-1018, April 1999.

20. R. H. Riedi and J. Lévy Véhel. Multifractal properties of TCP traffic: a numerical study. INRIA research report 3129, Rice University, February 1997.

21. M. S. Taqqu, V. Teverovsky, and W. Willinger. Is network traffic self-similar or multifractal? Fractals, 5:63-73, 1997.

22. J. Lévi Véhel and B. Sikdar. A multiplicative multifractal model for TCP traffic. In Proc., IEEE ISCC, Hammamet, Tunisia, July 2001. 